\title{
Cardiac injury in patients with COPD presenting with dyspnea: a pilot study
}

This article was published in the following Dove Press journal: International Journal of Chronic Obstructive Pulmonary Disease 8 November 2010

Number of times this article has been viewed

\author{
Esosa Odigie-Okon \\ Bryan Jordan \\ Sylvester Dijeh \\ Armand Wolff \\ Razvan Dadu \\ Priyanka Lall \\ Stuart Zarich \\ Yaw Amoateng-Adjepong \\ Constantine A Manthous \\ Bridgeport Hospital and Yale \\ University School of Medicine, \\ Bridgeport, CT, USA
}

Correspondence: Constantine A Manthous Bridgeport Hospital and Yale University School of Medicine, 267 Grant Street, Bridgeport, CT 06610, USA

Tel + I 203384458 I

Fax +I 2033844294

Email pcmant@bpthosp.org
Purpose: The aim of this pilot study was to test the hypothesis that myocardial ischemia complicates the management of some patients with chest-pain-free chronic obstructive pulmonary disease (COPD) exacerbations.

Methods: In this prospective, observational, cohort study, patients admitted to a 350-bed community teaching hospital, with dyspnea and a primary diagnosis of COPD exacerbation, were followed for enzymatic and electrocardiographic evidence of myocardial ischemia for the first 24 hours of hospital admission.

Results: A total of 114 patients were studied. Overall, four patients had definite myocardial infarctions, one had definite myocardial ischemia and 14 had possible myocardial ischemia. In multiple logistic regression models, age, number of coronary risk factors, and amount of administered albuterol were not associated with myocardial injury.

Conclusion: While unrecognized myocardial injury is relatively rare in patients with an exacerbation of COPD, it occurs frequently enough to warrant some caution since beta-agonists are the mainstays of therapy.

Keywords: COPD, myocardial ischemia, acute coronary syndrome, troponin

\section{Introduction}

Distinguishing dyspnea caused by heart versus lung disease has long been recognized as a very difficult task for chest and emergency physicians. ${ }^{1}$ Patients with chronic obstructive pulmonary disease (COPD) often present to hospital with acute dyspnea. These patients often have co-existent risks for - or established - coronary heart disease (CHD), which may mimic dyspnea related to COPD. The Global Initiative for Chronic Obstructive Lung Disease (GOLD) highlights that the population of patients with COPD also has common pathway comorbidities (ischemic heart disease and lung cancer); complicating comorbidities (pulmonary hypertension and heart failure); and coincidental or intercurrent comorbidities (eg, infections, dementia, depression), that may contribute substantially to acute or chronic illness. ${ }^{2}$

Since treatment of COPD exacerbations includes aerosolized beta-agonists that can exacerbate $\mathrm{CHD}$, it is incumbent on clinicians to detect dyspnea due to CHD versus COPD, to properly treat dyspnea. In a preliminary review of medical records, we reported that 26 of 27 patients discharged with a primary diagnosis of myocardial infarction (MI) and secondary diagnosis of COPD, who presented to our emergency department with dyspnea but not chest pain, had troponin-T elevations in the first 24 hours of their stay. ${ }^{3}$ Since not all COPD patients with acute dyspnea but no chest discomfort receive electrocardiographic (ECG) and enzymatic follow-up to rule-out acute coronary syndrome, 
we reasoned that some patients with cardiac-related dyspnea could be escaping detection. In this prospective study, we hypothesized that some patients with COPD presenting to hospital with dyspnea ascribed to COPD exacerbation, have unrecognized active CHD as the cause, or develop secondary CHD complicating their management.

\section{Methods}

\section{Study design and subjects}

This prospective cohort study was conducted after approval by the investigational review committee of Bridgeport Hospital, a 350-bed community teaching hospital affiliated with the Yale New Haven Health System. The Hospital Board waived requiring informed consent of patients owing to the minimal risk to patients. All patients aged greater than 50 years who were admitted to the hospital medical wards with the complaint of dyspnea, attributed by emergency department physicians to COPD exacerbation, from September 30th 2008 to April 30th 2009, were eligible for inclusion. Patients were excluded if they: 1) had chest pain; 2) were admitted for comfort care only; and/or 3) were admitted for "ruling out" acute coronary syndrome. All patients met GOLD criteria for COPD exacerbation, namely "an event in the natural course of the disease characterized by a change in the patient's baseline dyspnea, cough and/or sputum that is beyond normal day-to-day variations, is acute in onset, and may warrant a change in regular medication in a patient with underlying COPD". ${ }^{2}$ All patients carried a clinical diagnosis of COPD and were receiving outpatient treatments for COPD before hospital admission.

For patients with more than one hospitalization during the study period, data from only the first admission were included in analysis. Patients' caregivers, who provided all clinical management, were encouraged to order serum troponin-T and electrocardiograms on admission and 8-24 hours following admission.

\section{Data collection}

Demographic and clinical data were abstracted from medical records and included age, gender, race, co-morbidities, previous cardiac history, medications (before and during admission), laboratory data, and admission chest radiograph interpretations. Sequential ECGs of patients during the first 24 hours of admission (and prior to admission, when available) were interpreted in blinded fashion by a boardcertified cardiologist (SZ). After examining ECGs, the cardiologist was given corresponding troponin and same-day creatinine values and, applying consensus-based definitions, ${ }^{4}$ rendered diagnoses of: definite $\mathrm{MI}$ (when both ECG and enzymes were definitive); ischemia (ECG definitive with normal enzymes); possible ischemia (nonspecific ECG changes with normal troponins, or normal ECG with slightly elevated troponins in the presence of renal disease); or not MI or ischemia. A troponin value $>0.10 \mathrm{ng} \cdot \mathrm{mL}^{-1}$ (in the absence of elevated serum creatinine) is considered abnormal in our laboratory and was required for designating definite or possible MI.

Records of patients with definite or possible cardiac injury were examined in greater detail to ascertain whether they had any 6-dermatome symptoms (chest pain/pressure, shoulder pain, left arm pain, etc) either before or in the first 24 hours of admission.

\section{Statistical analyses}

Non-normally distributed variables are reported as median and interquartile ranges. Categorical variables are reported as percentages of total numbers of patients and comparisons are made using chi-square tests. The primary outcome of interest is the proportion of patients who suffer myocardial injury. Demographic and clinical data of these patients were compared to patients who had no evidence of myocardial infarction or ischemia. Logistic regression analyses were used to determine independent predictors of myocardial injury. Variables found to be significantly associated with myocardial injury in univariate analyses and those with biological plausibility were included in stepwise, backward elimination, multiple logistic regression models. Analyses were performed using Stata 9 software (StataCorp, College Station, TX). A two tailed $P$-value of $<0.05$ was considered statistically significant.

\section{Results}

A total of 114 patients were studied. Patients averaged 72.5 years (median 73 years; range 50-95 years); 59\% of 114 patients were female; and 70\% were caucasian (see Table 1). All patients had two or more (mean $4 \pm 1.4$ ) coronary risk factors and received an average of $14.3 \mathrm{mg}$ of aerosolized albuterol in the first 24 hours of treatment. Seventy patients had both initial and follow-up ECGs and troponin measurements; 76 had more than only one ECG; 99 had more than one troponin measurement; and 15 had only one troponin measurement. Overall, four patients had definite MI, one had definite ischemia, and 14 had possible ischemia. In the four patients with definite $\mathrm{MI}$, sequential troponin values were $0.02,0.14 ; 0.56,0.53$; $0.01,0.60$; and $0.14,0.10 \mathrm{ng} \cdot \mathrm{mL}^{-1}$ (see Figure 1). One patient with definite ischemia had sequential troponin values of 0.01 , $0.09 \mathrm{ng} \cdot \mathrm{mL}^{-1}$. Two patients with possible ischemia had values 
Table I Characteristics of studied patients*

\begin{tabular}{|c|c|c|c|c|}
\hline Variable & Total & $\begin{array}{l}\text { Ischemia/MI } \\
\text { n (\%) }\end{array}$ & $\begin{array}{l}\text { Nonischemia/MI } \\
\text { n (\%) }\end{array}$ & $P$-value \\
\hline & $N=114$ & $19(17)$ & $95(83)$ & \\
\hline Mean age $(S D)$ in years & $72.5(10)$ & $74.9(10)$ & $72.1(10)$ & 0.27 \\
\hline \multicolumn{5}{|l|}{ Gender } \\
\hline Female & 67 & $12(18)$ & $55(82)$ & \\
\hline Male & 47 & $7(15)$ & $40(85)$ & 0.67 \\
\hline \multicolumn{5}{|l|}{ Race } \\
\hline White & $80(70)$ & $14(18)$ & $66(83)$ & \\
\hline Nonwhite & $34(30)$ & $5(15)$ & $29(85)$ & 0.71 \\
\hline Mean number of coronary artery disease risk factors & 4.1 & 4.2 & 4.1 & 0.67 \\
\hline \multicolumn{5}{|l|}{ Comorbidities } \\
\hline Number of comorbidities & 4.3 & 4.4 & 4.2 & 0.67 \\
\hline Diabetes mellitus & 27 & $6(78)$ & $21(22)$ & 0.38 \\
\hline Hypertension & 66 & $9(14)$ & $57(86)$ & 0.31 \\
\hline Obesity & 50 & II (22) & $39(78)$ & 0.18 \\
\hline Coronary artery disease & 22 & $4(18)$ & $18(82)$ & 0.83 \\
\hline Hypercholesterolemia & 28 & $4(14)$ & $24(86)$ & 0.70 \\
\hline \multicolumn{5}{|l|}{ Vital signs } \\
\hline Heart rate on admission (beats/min) & 94 & 93 & 95 & 0.77 \\
\hline Respiratory rate (breaths/min) & 23 & 25 & 23 & 0.10 \\
\hline Systolic blood pressure on admission (mmhg) & 137 & $|4|$ & 136 & 0.49 \\
\hline Diastolic blood pressure on admission (mmhg) & 79 & 79 & 79 & 0.96 \\
\hline Creatinine $(\mathrm{mg} / \mathrm{dL})$ & 0.95 & 1.19 & 0.90 & $0.01 * *$ \\
\hline Cumulative albuterol (mg) & 14.3 & 15.1 & 14.1 & 0.72 \\
\hline Hospital length of stay (d) & 7.6 & 6.3 & 7.8 & 0.60 \\
\hline
\end{tabular}

Notes: *All patients had a positive smoking history. $* * P<0.05$.

Abbreviations: SD, standard deviation.

of $1.16,1.19$ and $0.11,0.11 \mathrm{ng} \cdot \mathrm{mL}^{-1}$. Thirty out of 53 patients who had BNP levels measured on admission had elevated levels $\left(>900 \mathrm{pg} \cdot \mathrm{mL}^{-1}\right)$. In both univariate analyses and multiple logistic regression models, age, number of coronary risk factors, chest radiographic consistent with congestive heart failure, and amount of administered albuterol were not associated with ischemia. Kidney dysfunction (creatinine $>1.2 \mathrm{mg} \cdot \mathrm{dL}^{-1}$ ), however, was significantly associated with ischemia in both univariate analyses (see Table 1) and multivariate modeling $(\mathrm{OR}=4.3$; 95\% CI: $1.2-15.3)$.

\section{Discussion}

To our knowledge, this is the first study to examine the rate at which unrecognized CHD either causes or complicates

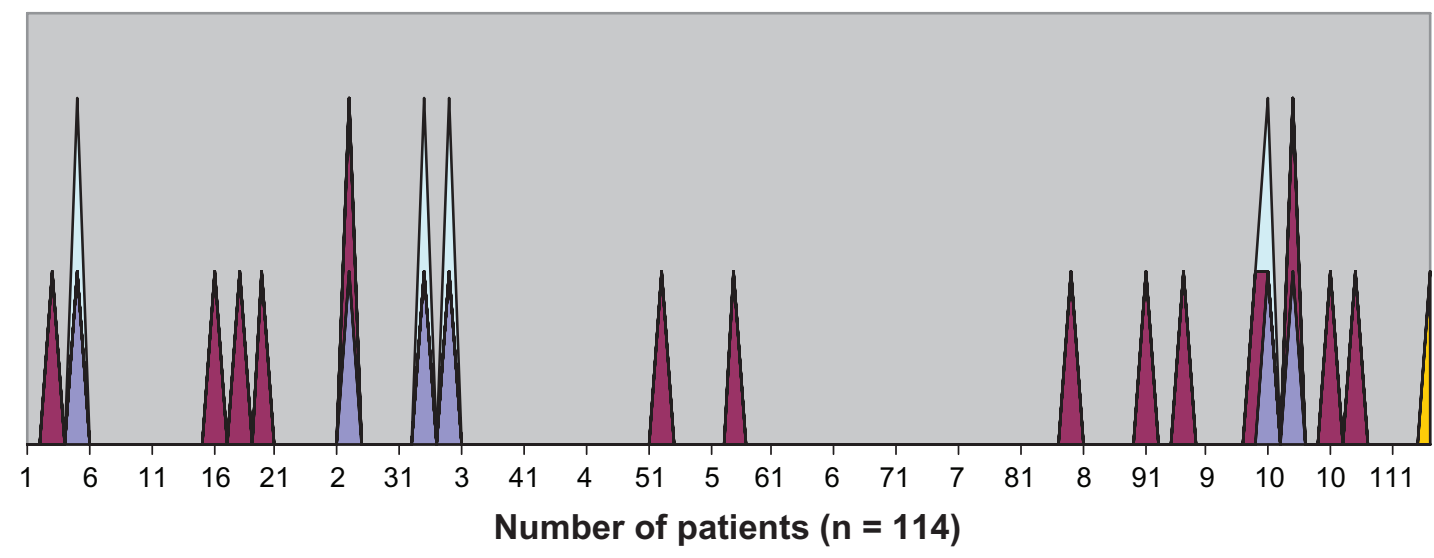

Positive troponin $\square$ Possible ischemia $\square$ Definite ischemia $\square$ Definite MI

Figure I Enzymatic and/or electrocardiographic evidence of myocardial injury (MI). 
the clinical course of COPD patients presenting to hospital with a complaint of dyspnea but no other symptoms of acute coronary syndrome. While the frequency of "definite" events was low (4\%), an additional 11\% had ECG and/or enzymatic evidence that was suggestive of myocardial injury. As serial ECGs and troponin determinations were not completed in all patients, this figure may underestimate the true prevalence. Even if only $4 \%$ of the over 700,000 patients admitted to hospital with COPD exacerbation each year ${ }^{5}$ have unrecognized myocardial injury, since such patients routinely receive beta-agonist therapy, this subset is at particular risk of unintended iatrogenic injury. ${ }^{6,7}$

COPD is a chronic inflammatory disease that promotes atherogenesis and CHD. ${ }^{8}$ During acute exacerbations, hypoxemia and stress associated with increased work of breathing may promote sympathetic overactivity ${ }^{9}$ that increases myocardial oxygen demand. Accordingly, myocardial ischemia and left ventricular dysfunction, which itself can promote wheezing, may complicate or be mistaken for an exacerbation of COPD. Our study cannot decipher whether ischemia is the primary reason for dyspnea, or a stress-related complication that contributes to dyspnea in this vulnerable cohort.

In a preliminary retrospective analysis, we reported that $29 \%$ of 94 patients with COPD discharged with a primary diagnosis of myocardial infarction, presented to hospital with dyspnea but no chest pain. Of these, 26 of 27 had troponin elevations in the first 24 hours of hospitalization and a majority $(62 \%)$ received beta-agonists aerosol therapies. ${ }^{3}$ Harvey and Hancox ${ }^{10}$ reported that 58 of 235 patients admitted to hospital with an exacerbation of COPD, who had troponin levels measured, had an elevated troponin. Seven of these were categorized as myocardial infarction. It is possible that troponin elevations associated with acute exacerbations of COPD arise from right heart strain associated with pulmonary hypertension. ${ }^{11}$ Additionally, even troponin elevations during COPD exacerbations do not necessarily connote classic acute coronary syndrome (ACS), since stress-related and/or therapeutically administered catecholamines may promote Tako-Tsubo phenomenon. ${ }^{12}$

Our study has a number of notable limitations. Firstly, the study protocol did not mandate that serial troponins and electrocardiograms be obtained for all patients. So, while 114 patients were studied, only 70 had sufficient data to determine whether they had myocardial injury. It is thus possible (or even likely, since those with chest pain thought to be coronary syndrome were excluded) that this study systematically underestimates the rate of cardiac ischemia complicating COPD exacerbation. Secondly, emergency physicians were aware of the study and its theoretical underpinnings and thus could have more vigorously scrutinized patients with COPD and CHD for ACS. This might also result in systematic underestimation of CHD complicating COPD. Cause and effect relationships cannot be inferred with our methodology. Additionally, the study is likely underpowered to detect potentially important pathophysiologic relationships (eg, albuterol dose and myocardial ischemia). Since inclusion was predicated on clinical grounds and not all patients had pulmonary function tests, it is possible that not all had COPD (despite being treated as outpatients for this diagnosis). Finally, there is no absolute gold standard for diagnosing myocardial ischemia in this population of patients. Patients with classic ACS symptoms were excluded, leaving only enzymatic and ECG evidence (European Society of Cardiology and American College of Cardiology criteria) for diagnosis. Electrocardiography in this demographic is highly nonspecific: while $11 \%$ had ECG signs that could connote ischemia, only $4 \%$ demonstrated either definitive changes or evolution with time to allow categorization of definite ischemia or non-ST elevation MI. Similarly, troponin values are highly sensitive but not very specific for detection of myocardial infarction in patients without classic ACS. Accordingly, we cannot exclude the possibility of some false positive results. Some bias could also have affected results, since emergency personnel were encouraged to obtain ECGs and troponins on patients in whom they previously had low (or no) clinical suspicion for ACS; we cannot discount the possibility that some patients were admitted with a primary diagnosis of myocardial injury as a result of our study. Since even mild renal dysfunction was independently associated with possible or definite myocardial injury, it is possible that some elevated troponins were "false positives", ie, not indicative of acute injury. Nonetheless, insofar as only two patients had isolated elevated troponin values (see Figure 1), this would impact our results minimally as renal dysfunction does not account for misclassification of ECGs. While a conservative interpretation of our results suggests only $4 \%$ of COPD patients presenting with dyspnea have either primary cardiac ischemia or cardiac ischemia complicating management, an additional $11 \%$ of patients had ECG evidence that could represent myocardial injury. Another weakness is that we did not ascertain the rate of myocardial ischemia in matched controls without COPD. But to the extent that otherwise clinically silent cardiac events may be "missed", this finding carries implications for management of between $4 \%$ and $15 \%$ of the over 700,000 patients each year who are hospitalized with COPD exacerbation, especially since beta-agonist aerosols may promote or exacerbate cardiac ischemia. ${ }^{6,7}$ 
In conclusion, this study demonstrates that ECG and enzymatic evidence of myocardial injury is relatively uncommon in patients presenting with acute exacerbations of COPD. Nonetheless, since beta-agonists are relatively contraindicated in patients with active cardiac ischemia, clinicians should be aware that a small subset of patients with COPD exacerbation may have primary or secondary cardiac ischemia that contributes to their dyspnea. Prospective studies that include patients with an acute exacerbation of (objectively-verified) COPD, ${ }^{2}$ and complete data (ie, serial ECG, troponin, and symptoms) would be required to definitively determine the frequency and clinical impact of this problem.

\section{Disclosure}

The authors have no financial or intellectual conflicts of interest relevant to this research. The research was not supported by external funding.

\section{References}

1. Logue RB, Hurst JW. Errors in the recognition and treatment of heart disease. Circulation. 1954;10:920-932.

2. Global Initiative for Chronic Obstructive Lung Disease. 2010. Available from: htttp://www.goldcopd.org/. Accessed Jun 8, 2010.
3. Parker H, Brenya R, Zarich S, Manthous CA. Beta-agonists for patients with chronic obstructive pulmonary disease and heart disease? Am J Emerg Med. 2008;26:104-105.

4. Thygesen K, Alpert JS, White HD, et al. Universal definition of myocardial infarction. Circulation. 2007;116:2634-2653.

5. Mannino DM, Homa DM, Akinbami LJ, Ford ES, Redd SC. Chronic obstructive pulmonary disease surveillance - United States, 1971-2000. MMWR Surveillance Summaries. 2002;51:1-16.

6. Gold DR, Zolli JR, Makaryus AN. Increased use of beta-agonists leading to non-ST segment elevation myocardial infarction in a patient with hemodynamically significant myocardial bridging. Mayo Clin Proc. 2008;83:956-960.

7. Seale JR, Wilkinson RS, Shanks SC, Watling J, Livingston WV. Myocardial infarction in status asthmaticus. Lancet. 1977;1: 658-659.

8. van Eeden SF, Sin DD. Chronic obstructive pulmonary disease: a chronic systemic inflammatory disease. Respiration. 2008;75:224-238.

9. Sakamaki F, Satoh T, Nagaya N, Kyotani S, Nakanishi N, Ishida Y. Abnormality of left ventricular sympathetic nervous function assessed by 123I-metaiodobenzylguanadine imaging in patients with COPD. Chest. 1999;116:1575-1581.

10. Harvey MG, Hancox RJ. Elevation of cardiac troponins in exacerbation of chronic obstructive pulmonary disease. Emergency Medicine Australasia. 2004;16:212-215.

11. Angulo F, Alvarado Y, Chokesuwattanaskul W, Roongsritong C. Troponin I elevation in a patient with severe bronchospasm. Am J Med Sci. 2005;329:320-321.

12. Hahn JY, Gwon HC, Park SW, et al. The clinical features of transient left ventricular nonapical ballooning syndrome: comparison with apical ballooning syndrome. Am Heart J. 2007;154:1166-1173.
International Journal of COPD

\section{Publish your work in this journal}

The International Journal of COPD is an international, peer-reviewed journal of therapeutics and pharmacology focusing on concise rapid reporting of clinical studies and reviews in COPD. Special focus is given to the pathophysiological processes underlying the disease, intervention programs, patient focused education, and self management protocols.

\section{Dovepress}

This journal is indexed on PubMed Central, MedLine and CAS. The manuscript management system is completely online and includes a very quick and fair peer-review system, which is all easy to use. Visit http://www.dovepress.com/testimonials.php to read real quotes from published authors. 